TRANSACTIONS OF THE

AMERICAN MATHEMATICAL SOCIETY

Volume 362, Number 9, September 2010, Pages 4497-4509

S 0002-9947(10)04734-3

Article electronically published on April 5, 2010

\title{
ISOPERIMETRIC INEQUALITIES FOR CONVEX HULLS AND RELATED QUESTIONS
}

\author{
PAOLO TILLI
}

\begin{abstract}
We consider the problem of maximizing the Lebesgue measure of the convex hull of a connected compact set of prescribed one-dimensional Hausdorff measure. In dimension two, we prove that the only solutions are semicircles. In higher dimensions, we prove some isoperimetric inequalities for convex hulls of connected sets; we focus on a classical open problem and discuss a possible new approach.
\end{abstract}

\section{INTRODUCTION}

In this paper we consider the problem, implicitly suggested in 3, of maximizing the volume of the convex hull of a connected compact set of given length in Euclidean space. Throughout the paper, the term "length" will always denote onedimensional Hausdorff measure $\mathcal{H}^{1}$, whereas the term "volume" stands for Lebesgue measure $\mathcal{L}^{d}$, where the dimension $d$ is that of the ambient space $\mathbb{R}^{d}$.

Thus, letting hull $(K)$ denote the convex hull of a set $K \subset \mathbb{R}^{d}$, our problem in its more general formulation can be stated as follows:

Problem 1.1. Given a real number $L>0$, find among all compact connected sets $K \subset \mathbb{R}^{d}$ such that $\mathcal{H}^{1}(K) \leq L$ those for which $\mathcal{L}^{d}(\operatorname{hull}(K))$ is a maximum.

Observe that the constraint that $\mathcal{H}^{1}(K) \leq L$ is equivalent to requiring equality, since if $\mathcal{H}^{1}(K)<L$, then a larger convex hull can be obtained by scaling. Hence, by homogeneity, our problem is equivalent to maximizing the isoperimetric ratio

$$
\frac{\mathcal{L}^{d}(\operatorname{hull}(K))}{\mathcal{H}^{1}(K)^{d}}
$$

among all connected compact sets such that $0<\mathcal{H}^{1}(K)<\infty$.

When $d=1$ the problem is trivial, hence we assume that $d>1$. The existence of solutions was proved by Gori [6] in any dimension, using the compactness and semicontinuity results of Blaschke and Gołab. However, finding the optimal sets seems to be a difficult problem.

In the particular case where $K$ is a closed curve, this problem was raised by Bonnesen and Fenchel [2] and, in dimension $d>2$, a complete solution is still missing. The problem is completely solved only in the case of curves that are convex in the sense of Schoenberg (i.e. curves in $\mathbb{R}^{d}$ which do not cross any hyperplane more than $d$ times; see [13]). For closed convex curves the problem was solved in [13, whereas for convex curves (not necessarily closed) it was solved by Nudel'man

Received by the editors November 22, 2006 and, in revised form, January 8, 2008.

2010 Mathematics Subject Classification. Primary 52A10, 52B60, 52A40.

(C) 2010 American Mathematical Society

Reverts to public domain 28 years from publication 
(see [7, 12]). It is not known, however, whether optimal convex curves are also optimal among all curves (this is a conjecture of Zalgaller; see [16]).

For curves in $\mathbb{R}^{3}$, either open or closed, solutions have been found only under extra symmetry assumptions (see [4, 8, 9,).

The planar case $d=2$ is easier. For closed curves, the problem is easily reduced to the standard isoperimetric inequality, whereas for open curves it is well known that the only solutions are semicircles (see e.g. 10]). It is also known how to locate in the plane a certain number of segments of prescribed lengths, in such a way that their union is a connected set and the area of their convex hull is a maximum (this problem was solved by A. Siegel; see [14, 15]).

In this paper we consider the more general case where $K$ is an arbitrary connected compact set (and not necessarily a union of segments, as was originally suggested in [3, p. 38) and we provide a full solution in the planar case.

Theorem 1.1. Assume $K \subset \mathbb{R}^{2}$ is a compact connected set, let $A$ denote the Lebesgue measure of its convex hull, and let $L=\mathcal{H}^{1}(K)$ denote its length which we assume to be finite. Then

$$
A \leq \frac{L^{2}}{2 \pi},
$$

and equality holds if and only if $K$ is a semicircle.

Problem 1.1 remains open in dimension $d>2$. However, we can prove the following bound for the volume of the convex hull.

Theorem 1.2. Assume $K \subset \mathbb{R}^{d}$ is a compact connected set such that $\mathcal{H}^{1}(K)$ is finite, and let $\nu: K \rightarrow S^{d-1}$ be a measurable selection of the unit tangent vector. Then

$$
\mathcal{L}^{d}(\operatorname{hull}(K)) \leq \frac{1}{(d !)^{2}} \int_{K} \ldots \int_{K}\left|\nu\left(x_{1}\right) \wedge \cdots \wedge \nu\left(x_{d}\right)\right| d \mathcal{H}^{1}\left(x_{1}\right) \cdots d \mathcal{H}^{1}\left(x_{d}\right) .
$$

As a consequence of the trivial bound $\left|\nu\left(x_{1}\right) \wedge \cdots \wedge \nu\left(x_{d}\right)\right| \leq 1$, one easily obtains the following corollary.

Corollary 1.3. Assume $K \subset \mathbb{R}^{d}$ is a compact connected set. Then

$$
\mathcal{L}^{d}(\operatorname{hull}(K)) \leq \frac{\mathcal{H}^{1}(K)^{d}}{(d !)^{2}}
$$

We point out that this inequality cannot be optimal in any dimension: in particular, in dimension two it should be replaced by (2) which is optimal.

Moreover, when $K$ is the image of a Lipschitz curve, the integrals in (3) can be parameterized, and one obtains the following corollary.

Corollary 1.4. Let $\gamma:[a, b] \rightarrow \mathbb{R}^{d}$ be a Lipschitz curve. Then

$$
V \leq \frac{1}{(d !)^{2}} \int_{a}^{b} \cdots \int_{a}^{b}\left|\gamma^{\prime}\left(t_{1}\right) \wedge \cdots \wedge \gamma^{\prime}\left(t_{d}\right)\right| d t_{1} \cdots d t_{d},
$$

where $V$ denotes the volume of the convex hull of $\gamma$.

In order to prove Theorem 1.1, in Section 3 we associate with every connected compact set $K \subset \mathbb{R}^{d}$ of finite length a Borel measure $\mu$ on the unit sphere $S^{d-1}$. This measure $\mu$ satisfies $\mu\left(S^{d-1}\right)=\mathcal{H}^{1}(K)$ and provides information on how the tangent space to $K$ is distributed on the unit sphere, up to orientation. For this 
reason, we call $\mu$ the direction measure associated to $K$. In principle, our approach to Problem 1.1 using the direction measure is valid in any dimension, and a complete solution of Problem 1.1 might be related to the following variational problem for measures.

Problem 1.2. In dimension $d \geq 3$, compute

$$
\theta(d):=\max _{\mu} \int_{S^{d-1}} \cdots \int_{S^{d-1}}\left|x_{1} \wedge \cdots \wedge x_{d}\right| d \mu\left(x_{1}\right) \cdots d \mu\left(x_{d}\right),
$$

where the maximum is over all probability measures $\mu$ on the unit sphere $S^{d-1}$, and find all measures for which the maximum is attained.

Note that, as the integrand function is even in each variable, one can restrict (5) to the case where the measure $\mu$ is even. The quantity (5) can be used to bound the isoperimetric ratio, as follows.

Theorem 1.5. For every $d \geq 2$ and every connected compact set $K \subset \mathbb{R}^{d}$ with $0<\mathcal{H}^{1}(K)<\infty$,

$$
\frac{\mathcal{L}^{d}(\mathrm{hull}(K))}{\mathcal{H}^{1}(K)^{d}} \leq \frac{\theta(d)}{(d !)^{2}}
$$

holds, where $\theta(d)$ is given by (5).

We believe that this bound might be sharp in any dimension. Indeed, when $d=2$, we can solve (5) explicitly (Theorem 4.1), with the resulting bound attained by the semicircle, and this allows us to prove (2) (uniqueness of the semicircle, however, is more delicate and requires additional work). Unfortunately, when $d>2$ we were not able to compute (5).

When $d=3$, it was proved by Nudel'man [7, 12 that among all convex curves, the isoperimetric ratio (1) is maximized by the helical curve

$$
x(t)=\cos t, \quad y(t)=\sin t, \quad z(t)=t / \sqrt{2}, \quad 0 \leq t \leq 2 \pi
$$

(which is the unique maximizer, up to similarity), and it has been conjectured [16] that this curve maximizes (10) among all curves, not necessarily convex. This motivates the following question.

Problem 1.3. Prove or disprove the following statement: when $d=3$, the maximum in (5) is achieved by the probability measure $\mu_{0}$ on the sphere $S^{2}$, given by Hausdorff measure $\mathcal{H}^{1}$ restricted to the two parallels

$$
\left\{(x, y, z) \in S^{2} \quad \text { such that }|z|=\frac{1}{\sqrt{3}}\right\},
$$

normalized to have total mass one.

We point out that the probability measure $\mu_{0}$ described above is obtained as the direction measure (see Section 3) relative to Nudel'man's helix (7) (normalized to have unitary length). An explicit computation reveals that

$$
\int_{S^{2}} \int_{S^{2}} \int_{S^{2}}|x \wedge y \wedge z| d \mu_{0}(x) d \mu_{0}(y) d \mu_{0}(z)=\frac{2}{\sqrt{3} \pi},
$$

and this quantity divided by $(d !)^{2}=36$ is exactly the isoperimetric ratio of the helical curve (7). Therefore, if the answer to Problem 1.3 were affirmative, it would follow that $\theta(3)=2 /(\sqrt{3} \pi)$ and hence, by ([6) , the helical curve (7) would be optimal 
among all connected compact sets, thus proving, in particular, the aforementioned conjecture of Zalgaller.

Note added in proof. The answer to Problem 1.3 turns out to be negative (for instance, the uniform measure on the sphere is a better competitor than the measure $\mu_{0}$ described above, which therefore does not yield the maximum in (5)). However, Problem 1.2 is still open, and an explicit solution would turn (6) into an effective bound, stronger than the bound in (4).

The paper is organized as follows. In Section 2 we prove Theorem 1.2, whereas in Section 3 we introduce the notion of direction measure and prove Theorem [1.5. Finally, Section 4 is devoted to the proof of Theorem 1.1

\section{A BOUND FOR THE VOLUME OF THE CONVEX HULL}

This section is devoted to the proof of Theorem [1.2 Our starting point is the following result of Gori, the proof of which can be found in [6].

Theorem 2.1 (Gori). Assume $K \subset \mathbb{R}^{d}$ is a connected compact set which can be written as the union of $n$ segments $(n \geq d)$ with endpoints $x_{i}, y_{i}, i=1, \ldots, n$. Then, letting $v_{i}=y_{i}-x_{i}$, we have

$$
\mathcal{L}^{d}(\operatorname{hull}(K)) \leq \frac{1}{d !} \sum_{1 \leq j_{1}<j_{2}<\cdots<j_{d} \leq n}\left|v_{j_{1}} \wedge \cdots \wedge v_{j_{d}}\right|
$$

In order to prove Theorem 1.2, we also need as a tool the following structure theorem for connected compact sets of finite length. The proof of this lemma can be found in [1] (see Theorem 4.4.8 therein and its proof).

Lemma 2.2. Assume that $K \subset \mathbb{R}^{d}$ is a compact connected set such that $\mathcal{H}^{1}(K)<$ $\infty$. Then there exists a countable family of Lipschitz curves $\gamma_{i}:\left[0, L_{i}\right] \rightarrow K, L_{i} \geq 0$, such that:

(1) Each $\gamma_{i}$ is injective and parameterized by arc length.

(2) Letting $K_{i}=\gamma_{i}\left(\left[0, L_{i}\right]\right)$, we have

$$
\mathcal{H}^{1}\left(K \backslash \bigcup_{i=1}^{\infty} K_{i}\right)=0
$$

(3) For every $j \geq 2$,

$$
K_{j} \cap \bigcup_{i=1}^{j-1} K_{i}=\left\{\gamma_{j}(0)\right\} .
$$

(4) The compact sets $\bigcup_{i=1}^{n} K_{i}$ converge to $K$ with respect to the Hausdorff distance, as $n \rightarrow \infty$.

It is well known that a compact connected set $K$ such that $\mathcal{H}^{1}(K)<\infty$ is rectifiable (indeed, this follows as a byproduct of the previous lemma, which can be seen as a strong rectifiability theorem). Thus $K$ has a tangent space (in the sense of geometric measure theory; see for instance [5]) at $\mathcal{H}^{1}$-a.e. point $x \in K$, and one can find a measurable map $\nu: K \rightarrow S^{d-1}$ such that for a.e. $x \in K$, the tangent space at $x$ is parallel to $\nu(x)$ (the construction of such maps can be achieved as a consequence of Lemma 2.2). Every such map $\nu$ is called a measurable selection of the tangent space (note that a tangent vector, where it exists, is unique up to orientation, hence the need for a measurable selection).

Now we are in a position to prove Theorem 1.2 . 
Proof of Theorem 1.2. Clearly we may assume that $K$ does not reduce to a single point. The actual proof is divided into several steps.

Step 1. First, we assume that $K$ is the union of $n$ segments, with endpoints $x_{i} \neq y_{i}, i=1, \ldots, n$. Decreasing if necessary the number of segments (i.e. dropping redundant segments and merging into a single segment any two collinear segments that are not disjoint), we may assume that any two segments have at most one point in common. In this case, the tangent vector is defined (up to orientation) at all points $x \in K$, except any that happen to be an endpoint of a segment or a possible intersection point of two or more segments. Thus, a measurable selection of the tangent vector $\nu(x)$ is given by

$$
\nu(x)=\frac{y_{i}-x_{i}}{\left|y_{i}-x_{i}\right|}
$$

if $x$ belongs to the segment from $x_{i}$ to $y_{i}$ (we may regard $\nu(x)$ as undefined if $x$ belongs to more than one segment).

If $n<d$, then clearly hull $(K)$ has zero measure. However, in this case the integral in (3) is zero as well, since for every choice of points $x_{1}, \ldots, x_{d}$ where $\nu$ is defined, at least two of them (say $x_{1}$ and $x_{2}$ ) belong to the same segment; hence $\nu\left(x_{1}\right)=\nu\left(x_{2}\right)$, and thus the wedge product under the integral sign vanishes almost everywhere. Hence (3) is trivial when $n<d$.

Now suppose that $n \geq d$. In this case, we claim that (3) reduces to (8). Let $s_{i} \subset K$ denote the segment with endpoints $x_{i}, y_{i}$, set $v_{i}=y_{i}-v_{i}$ and let $\nu_{i}=v_{i} /\left|v_{i}\right|$ be the tangent vector along the segment $s_{i}$. Recall that any two segments have at most one point in common, thus overlapping is $\mathcal{H}^{1}$-negligible. Hence we can split each nested integral into a sum of integrals over each single segment, obtaining

$$
\begin{gathered}
\int_{K} \ldots \int_{K}\left|\nu\left(x_{1}\right) \wedge \cdots \wedge \nu\left(x_{d}\right)\right| d \mathcal{H}^{1}\left(x_{1}\right) \cdots d \mathcal{H}^{1}\left(x_{d}\right) \\
=\sum_{j_{1}=1}^{n} \cdots \sum_{j_{d}=1}^{n} \int_{s_{j_{1}}} \ldots \int_{s_{j_{d}}}\left|\nu\left(x_{1}\right) \wedge \cdots \wedge \nu\left(x_{d}\right)\right| d \mathcal{H}^{1}\left(x_{1}\right) \cdots d \mathcal{H}^{1}\left(x_{d}\right) \\
=\sum_{j_{1}=1}^{n} \cdots \sum_{j_{d}=1}^{n} \int_{s_{j_{1}}} \cdots \int_{s_{j_{d}}}\left|\nu_{j_{1}} \wedge \cdots \wedge \nu_{j_{d}}\right| d \mathcal{H}^{1}\left(x_{1}\right) \cdots d \mathcal{H}^{1}\left(x_{d}\right) \\
=\sum_{j_{1}=1}^{n} \cdots \sum_{j_{d}=1}^{n} \mathcal{H}^{1}\left(s_{j_{1}}\right) \times \cdots \times \mathcal{H}^{1}\left(s_{j_{d}}\right) \times\left|\nu_{j_{1}} \wedge \cdots \wedge \nu_{j_{d}}\right| \\
=\sum_{j_{1}=1}^{n} \cdots \sum_{j_{d}=1}^{n}\left|v_{j_{1}} \wedge \cdots \wedge v_{j_{d}}\right|,
\end{gathered}
$$

where for the last equality we have used the fact that $\mathcal{H}^{1}\left(s_{j}\right) \nu_{j}=v_{j}$. Then, using the invariance of the summand function with respect to permutations and neglecting all $d$-tuples where any two subscripts are equal (for which the wedge product vanishes), we finally obtain

$$
\begin{gathered}
\int_{K} \ldots \int_{K}\left|\nu\left(x_{1}\right) \wedge \cdots \wedge \nu\left(x_{d}\right)\right| d \mathcal{H}^{1}\left(x_{1}\right) \cdots d \mathcal{H}^{1}\left(x_{d}\right)= \\
d ! \sum_{1 \leq j_{1}<\cdots<j_{d} \leq n}\left|v_{j_{1}} \wedge \cdots \wedge v_{j_{d}}\right|,
\end{gathered}
$$

i.e. the right hand side of (3) is equal to that of (8). 
Step 2. Here we assume that $K$ is the image of an injective Lipschitz curve $\gamma:[0, L] \rightarrow \mathbb{R}^{d}$, parameterized by arc length (hence $\left|\gamma^{\prime}(t)\right|=1$ for a.e. $t$ ). Now consider a sequence of polygonal curves $\gamma_{n}:[0, L] \rightarrow \mathbb{R}^{d}$ (i.e. continuous and piecewise affine maps) such that $\gamma_{n} \rightarrow \gamma$ uniformly and $\gamma_{n}^{\prime} \rightarrow \gamma^{\prime}$ pointwise almost everywhere, as $n$ tends to infinity. For instance, one can construct $\gamma_{n}$ as follows:

$$
\begin{aligned}
& \gamma_{n}(0)=\gamma(0), \\
& \gamma_{n}^{\prime}(t)=\frac{L}{n} \int_{j L / n}^{(j+1) L / n} \gamma^{\prime}(s) d s \quad \text { if } \quad \frac{j L}{n}<t<\frac{(j+1) L}{n}, j=0, \ldots, n-1 .
\end{aligned}
$$

Note that each $\gamma_{n}$ is a polygonal curve which interpolates $\gamma$ at the points $j L / n$. Uniform convergence is clear, whereas pointwise convergence of derivatives a.e. follows from the Lebesgue Theorem. In particular, letting $K_{n}=\gamma_{n}([0, L])$, we have $K_{n} \rightarrow K$ in Hausdorff distance, hence (see [6])

$$
\lim _{n \rightarrow \infty} \mathcal{L}^{d}\left(\operatorname{hull}\left(K_{n}\right)\right)=\mathcal{L}^{d}(\operatorname{hull}(K)) .
$$

Moreover, as each $K_{n}$ is a finite union of segments, letting $\nu_{n}(x)$ denote the unit tangent vector at $x \in K_{n}$ we have that

$$
\mathcal{L}^{d}\left(\operatorname{hull}\left(K_{n}\right)\right) \leq \frac{1}{(d !)^{2}} \int_{K_{n}} \ldots \int_{K_{n}}\left|\nu_{n}\left(x_{1}\right) \wedge \cdots \wedge \nu_{n}\left(x_{d}\right)\right| d \mathcal{H}^{1}\left(x_{1}\right) \cdots d \mathcal{H}^{1}\left(x_{d}\right)
$$

by Step 1 of this proof. Note that $\gamma_{n}$ need not be injective; however, for all $x \in K_{n}$ except possibly segment endpoints or crossing points of two segments (i.e. apart from finitely many exceptions) we clearly have

$$
\text { either } \nu_{n}(x)=\frac{\gamma_{n}^{\prime}(t)}{\left|\gamma_{n}^{\prime}(t)\right|} \text { or } \quad \nu_{n}(x)=-\frac{\gamma_{n}^{\prime}(t)}{\left|\gamma_{n}^{\prime}(t)\right|}
$$

for every $t$ such that $\gamma_{n}(t)=x$ (note that $\gamma_{n}^{\prime}(t) \neq 0$ for a.e. $t$ by construction, since $\gamma$ is injective). Therefore, since the integrand function is nonnegative, we have by the area formula that

$$
\begin{gathered}
\int_{K_{n}} \cdots \int_{K_{n}}\left|\nu_{n}\left(x_{1}\right) \wedge \cdots \wedge \nu_{n}\left(x_{d}\right)\right| d \mathcal{H}^{1}\left(x_{1}\right) \cdots d \mathcal{H}^{1}\left(x_{d}\right) \\
\leq \quad \int_{0}^{L} \cdots \int_{0}^{L}\left|\frac{\gamma_{n}^{\prime}\left(t_{1}\right)}{\left|\gamma_{n}^{\prime}\left(t_{1}\right)\right|} \wedge \cdots \wedge \frac{\gamma_{n}^{\prime}\left(t_{d}\right)}{\left|\gamma_{n}^{\prime}\left(t_{d}\right)\right|}\right| \times\left|\gamma_{n}^{\prime}\left(t_{1}\right)\right| \times \cdots \times\left|\gamma_{n}^{\prime}\left(t_{d}\right)\right| d t_{1} \cdots d t_{d}
\end{gathered}
$$

(in order to have equality, one should insert the cardinality of $\left\{\gamma_{n}^{-1}\left(x_{i}\right)\right\}$ in each nested integral in the left hand side, i.e. consider multiplicities).

Now, letting $n \rightarrow \infty$, the last integral converges (by dominated convergence, recalling that $\gamma_{n}^{\prime} \rightarrow \gamma^{\prime}$ pointwise a.e.) to the corresponding integral relative to $\gamma$; hence, on combining the last two inequalities and passing to the limit, by recalling (9) we find that

$$
\mathcal{L}^{d}(\operatorname{hull}(K)) \leq \frac{1}{(d !)^{2}} \int_{0}^{L} \cdots \int_{0}^{L}\left|\gamma^{\prime}\left(t_{1}\right) \wedge \cdots \wedge \gamma^{\prime}\left(t_{d}\right)\right| d t_{1} \cdots d t_{d}
$$

where we have used the fact that $\left|\gamma^{\prime}(t)\right|=1$ for a.e. $t$. On the other hand, since $\gamma$ is injective and $\left|\gamma^{\prime}(t)\right|=1$, by the area formula the last integral equals the integral in (3), which is therefore established when $K$ is the image of an injective Lipschitz curve. 
Step 3. Using exactly the same technique as in the previous step, one can prove (3) when $K$ is the union of finitely many Lipschitz curves. More precisely, assume that, for some $m \geq 2$, there exist Lipschitz maps $\Gamma_{i}:\left[0, L_{i}\right] \rightarrow K, i=1, \ldots, m$, such that

(1) Each $\Gamma_{i}$ is injective and parameterized by arc length.

(2) $K=\bigcup_{i=1}^{m} K_{i}$, where $K_{i}=\Gamma_{i}\left(\left[0, L_{i}\right]\right)$ is the image of $\Gamma_{i}$.

(3) For every $j$ with $2 \leq j \leq m$,

$$
K_{j} \cap \bigcup_{i=1}^{j-1} K_{i}=\left\{\Gamma_{j}(0)\right\} .
$$

Intuitively, each simple curve $K_{i}$ represents a branch of $K$, and each new branch $K_{j}$ is attached to the previous ones by its starting point $\Gamma_{j}(0)$ (and by no other point). In order to prove (3), it suffices to approximate $K$ by the union of $m$ polygonal curves, each obtained by interpolating (at uniform nodes on $\left[0, L_{i}\right]$ ) the corresponding branch $\Gamma_{i}$ as in Step 2, and pass to the limit. The only issue for which some care is needed is the concern that if one interpolates each branch of $K$ by a polygonal curve independently of other branches, then the union of these $m$ polygonal curves at some stage might fail to be a connected set (and hence one could not invoke Step 1). On the other hand, one can easily overcome this difficulty by adding extra interpolation points: indeed, it suffices to require that for all $i<j$ such that $K_{j} \cap K_{i} \neq \emptyset$ (this occurs when the branch $K_{j}$ is attached to $K_{i}$ ), the (unique by property 3 ) junction point $\Gamma_{j}(0)$ should be an interpolation point for the curve $\Gamma_{i}$. Then the proof is the same as in Step 2, with only notational changes, and the details are left to the reader.

Step 4. Finally, we consider the general case where $K$ is a connected compact set such that $\mathcal{H}^{1}(K)<\infty$. Let $K_{i}$ be as in Lemma 2.2 and set

$$
C_{n}=\bigcup_{i=1}^{n} K_{i}
$$

Then, by Lemma 2.2, each $C_{n}$ is a finite union of Lipschitz curves, meeting the requirement of Step 3 of this proof. Then, by Step 3, we have

$$
\begin{aligned}
\mathcal{L}^{d}\left(\operatorname{hull}\left(C_{n}\right)\right) \leq & \frac{1}{(d !)^{2}} \int_{C_{n}} \ldots \int_{C_{n}}\left|\nu_{n}\left(x_{1}\right) \wedge \ldots \wedge \nu_{n}\left(x_{d}\right)\right| d \mathcal{H}^{1}\left(x_{1}\right) \cdots d \mathcal{H}^{1}\left(x_{d}\right) \\
& \leq \frac{1}{(d !)^{2}} \int_{K} \ldots \int_{K}\left|\nu\left(x_{1}\right) \wedge \ldots \wedge \nu\left(x_{d}\right)\right| d \mathcal{H}^{1}\left(x_{1}\right) \cdots d \mathcal{H}^{1}\left(x_{d}\right)
\end{aligned}
$$

where $\nu_{n}: C_{n} \rightarrow S^{d-1}$ and $\nu: K \rightarrow S^{d-1}$ are measurable selections of the tangent vectors (it is well known that, since $C_{n} \subseteq K, \nu_{n}(x)=\nu(x)$ for a.e. $x \in C_{n}$; hence the last inequality follows from the inclusion $\left.C_{n} \subseteq K\right)$. Finally, taking the limit as $n \rightarrow \infty$ in the last estimate, the first term tends to $\mathcal{L}^{d}(\operatorname{hull}(K)$ ) (by statement 4 of Lemma 2.2), and (3) follows.

\section{The DiRECTION MEASURE}

In this section we introduce a geometric tool which will be useful in what follows. Our goal is to associate with every compact connected set $K \subset \mathbb{R}^{d}$ of finite length a finite Borel measure $\mu$ on the unit sphere $S^{d-1}$, which carries information on how the tangent vectors are distributed on the unit sphere. 
Definition 3.1. Assume $K \subset \mathbb{R}^{d}$ is a compact connected set such that $\mathcal{H}^{1}(K)<$ $\infty$, and let $\nu: K \rightarrow S^{d-1}$ be a measurable selection of the unit tangent vector. For every Borel set $E \subseteq S^{d-1}$, let

$$
\lambda(E):=\mathcal{H}^{1}(\{x \in K \mid \nu(x) \in E\})
$$

and

$$
\mu(E)=\frac{\lambda(E)+\lambda(-E)}{2},
$$

where $-E=\{x \mid-x \in E\}$ is the symmetric set of $E$ with respect to the origin. We say that $\mu$ is the direction measure of $K$.

Some remarks are in order. It is clear that the auxiliary map $\lambda$ defined by (10) is a Borel measure on the unit sphere such that $\lambda\left(S^{d-1}\right)=\mathcal{H}^{1}(K)$, hence the direction measure $\mu$ also has these properties.

Note that $\lambda$ depends on (the orientation of) the tangent field $\nu$; to get rid of this dependence, we define the direction measure $\mu$ from $\lambda$ by symmetrization with respect to the origin, according to (11). Since, given two measurable selections $\nu_{1}$, $\nu_{2}$ of the tangent field, we clearly have $\nu_{1}(x)= \pm \nu_{2}(x)$ for $\mathcal{H}^{1}$-a.e. $x \in K$, it is clear that the measure $\mu$ depends only on $K$ and not on $\nu$.

Of course, one could also regard $\mu$ as a measure on the real projective space, but working with a symmetric measure on the sphere, rather than a measure on the projective space, is more convenient for our purposes here.

Note that (10) is equivalent to the condition that

$$
\int_{S^{d-1}} h(y) d \lambda(y)=\int_{K} h(\nu(x)) d \mathcal{H}^{1}(x)
$$

for every nonnegative Borel function $h$. As a consequence, we have

$$
\int_{S^{d-1}} h(y) d \mu(y)=\frac{1}{2} \int_{K}(h(\nu(x))+h(-\nu(x))) d \mathcal{H}^{1}(x)
$$

for every nonnegative Borel function $h$ or, equivalently,

$$
\int_{S^{d-1}} h(y) d \mu(y)=\int_{K} h(\nu(x)) d \mathcal{H}^{1}(x)
$$

for every nonnegative Borel function $h$ such that $h(y)=h(-y)$.

Using the direction measure, we can rewrite the integral in (3), on the cartesian product $K \times \cdots \times K$, as an integral on the cartesian product $S^{d-1} \times \cdots \times S^{d-1}$, allowing us to pass from a problem where the unknown is a set to a problem where the unknown is a measure. Indeed, we have the following:

Proposition 3.2. Assume $K \subset \mathbb{R}^{d}$ is a compact connected set such that $\mathcal{H}^{1}(K)<$ $\infty$, and let $\mu$ be the direction measure associated to $K$. Then

$$
\mathcal{L}^{d}(\operatorname{hull}(K)) \leq \frac{1}{(d !)^{2}} \int_{S^{d-1}} \cdots \int_{S^{d-1}}\left|y_{1} \wedge \cdots \wedge y_{d}\right| d \mu\left(y_{1}\right) \cdots d \mu\left(y_{d}\right) .
$$

Proof. Let $\nu: K \rightarrow S^{d-1}$ be a measurable selection of the tangent vector. Then, for every $d-1$ points $x_{2}, \ldots, x_{d}$ in $K$ where $\nu$ is defined, the function

$$
h(y)=\left|y \wedge \nu\left(x_{2}\right) \wedge \cdots \wedge \nu\left(x_{d}\right)\right|
$$


satisfies $h(y)=h(-y)$. Hence from (12) we find that

$$
\begin{array}{r}
\int_{K} \cdots \int_{K}\left|\nu\left(x_{1}\right) \wedge \cdots \wedge \nu\left(x_{d}\right)\right| d \mathcal{H}^{1}\left(x_{1}\right) \cdots d \mathcal{H}^{1}\left(x_{d}\right) \\
=\int_{K} \cdots \int_{K}\left(\int_{S^{d-1}}\left|y_{1} \wedge \nu\left(x_{2}\right) \wedge \cdots \wedge \nu\left(x_{d}\right)\right| d \mu\left(y_{1}\right)\right) d \mathcal{H}^{1}\left(x_{2}\right) \cdots d \mathcal{H}^{1}\left(x_{d}\right) .
\end{array}
$$

Proceeding in this way for each of the other nested integrals, one obtains (13) from (3).

Now, the proof of Theorem 1.5 is straightforward.

Proof of Theorem 1.5. Let $K$ be as in Theorem 1.5. By homogeneity, we can assume that $\mathcal{H}^{1}(K)=1$; hence the direction measure $\mu$ associated to $K$ is a probability measure on the unit sphere. Then (6) follows immediately from Proposition 3.2 and (5).

\section{The Planar CASE}

In this section we prove Theorem [1.1 thus solving Problem 1.1 in the planar case. The first step is to characterize all even measures $\mu$ which attain (5) when $d=2$.

Theorem 4.1. Let $\mu$ be a Borel measure on the unit circle $S^{1}$. Suppose $\mu$ is even, i.e.

$$
\mu(E)=\mu(-E) \quad \text { for every Borel set } E \subseteq S^{1} .
$$

Then

$$
\int_{S^{1}} \int_{S_{1}}|x \wedge y| d \mu(x) d \mu(y) \leq \frac{2}{\pi} \mu\left(S^{1}\right)^{2}
$$

and equality occurs if and only if $\mu$ is a multiple of Lebesgue measure.

Proof. We can identify $S^{1}$ with the interval $[0,2 \pi)$ and, using angular variables $x$ and $y$, the integral in question can be written as

$$
\int_{0}^{2 \pi} \int_{0}^{2 \pi}|\sin (y-x)| d \mu(x) d \mu(y)
$$

where $\mu$ is now regarded as a Borel measure on $\mathbb{R}$, periodic with period $\pi$ and satisfying (14). Consider the Fourier coefficients of $\mu$, given by

$$
a_{j}=\frac{1}{2 \pi} \int_{0}^{2 \pi} e^{-1 j x} d \mu(x), \quad j \in \mathbb{Z} .
$$

Since $\mu$ is a real measure with period $\pi$, we have

$$
a_{-j}=\overline{a_{j}}, \quad a_{2 j+1}=0 \quad \forall z \in \mathbb{Z} .
$$

Similarly, if

$$
|\sin t|=\sum_{j=-\infty}^{\infty} b_{j} e^{1 j t}
$$

is the Fourier series of $|\sin t|$, we have

$$
b_{-j}=\overline{b_{j}} \in \mathbb{R}, \quad b_{2 j+1}=0 \quad \forall z \in \mathbb{Z}
$$


since $|\sin t|$ is even, real, and $\pi$-periodic. Note that

$$
a_{0}=\frac{\mu\left(S^{1}\right)}{2 \pi}, \quad b_{0}=\frac{1}{2 \pi} \int_{0}^{2 \pi}|\sin t| d t=\frac{2}{\pi} .
$$

Then, plugging the Fourier expansion (18) (which converges uniformly) into (16) and using (17), (19) and (20), we obtain

$$
\begin{array}{r}
\int_{0}^{2 \pi} \int_{0}^{2 \pi}|\sin (y-x)| d \mu(x) d \mu(y)=\sum_{j=-\infty}^{\infty} b_{j} \int_{0}^{2 \pi} \int_{0}^{2 \pi} e^{1 j y} e^{-1 j x} d \mu(x) d \mu(y)= \\
=4 \pi^{2} \sum_{j=-\infty}^{\infty} b_{j} a_{-j} a_{j}=4 \pi^{2} \sum_{j=-\infty}^{\infty} b_{j}\left|a_{j}\right|^{2}=4 \pi^{2} b_{0}\left|a_{0}\right|^{2}+8 \pi^{2} \sum_{j=1}^{\infty} b_{2 j}\left|a_{2 j}\right|^{2} \\
=\frac{2}{\pi} \mu\left(S^{1}\right)^{2}+8 \pi^{2} \sum_{j=1}^{\infty} b_{2 j}\left|a_{2 j}\right|^{2} .
\end{array}
$$

On the other hand, the Fourier expansion (18) is well known, and one has

$$
b_{2 j}=\frac{-4}{\pi\left(4 j^{2}-1\right)} \quad \forall j \geq 1 .
$$

Therefore, (15) follows since $b_{2 j}<0$ for $j \geq 1$. Moreover, if equality holds in (15), then necessarily

$$
\sum_{j=1}^{\infty} b_{2 j}\left|a_{2 j}\right|^{2}=0,
$$

hence $a_{2 j}=0$ for every $j \geq 1$. This condition, combined with (17), means that $a_{j}=0$ for every $j$, except possibly for $j=0$, i.e. $\mu$ is a multiple of Lebesgue measure.

Now we are in a position to prove Theorem 1.1 .

Proof of Theorem 1.1, Let $K \subset \mathbb{R}^{2}$ be a connected compact set with $L=\mathcal{H}^{1}(K)<$ $\infty$, and let $\mu$ be its direction measure. Proposition 3.2 yields the bound

$$
\mathcal{L}^{2}(\operatorname{hull}(K)) \leq \frac{1}{4} \int_{S^{1}} \int_{S_{1}}|x \wedge y| d \mu(x) d \mu(y) ;
$$

hence, recalling that $\mu\left(S^{1}\right)=\mathcal{H}^{1}(K)$, Theorem 4.1 gives

$$
\mathcal{L}^{2}(\operatorname{hull}(K)) \leq \frac{\mathcal{H}^{1}(K)^{2}}{2 \pi}
$$

which is the desired inequality (2). Now suppose that equality holds for a given $K$. Then equality must hold in (15), and from Theorem 4.1 we obtain that $\mu$ is a multiple of Lebesgue measure (in particular, $\mu$ has no atoms).

We have to prove that $K$ is a semicircle. First we claim that

$$
K \subset \partial \operatorname{hull}(K) \text {, }
$$

i.e. $K$ is entirely contained in the boundary of its convex hull. For this purpose, we assume that there exists some $x_{0} \in K$ which is internal to hull $(K)$ and seek a contradiction.

Since $\mathcal{H}^{1}(K)$ is finite, it is well known that for almost every $r>0$ the intersection of $K$ with the boundary of the ball $B\left(x_{0}, r\right)$ is made up of finitely many points (otherwise, upon slicing $K$ with circles centred at $x_{0}$, by the coarea formula we 
would obtain that $\mathcal{H}^{1}(K)=\infty$, a contradiction). In particular, we can find some small $r>0$ such that $\overline{B\left(x_{0}, r\right)}$ is in the interior of hull $(K)$ and $K \cap \partial B\left(x_{0}, r\right)$ is a finite set. Now let $S$ denote the connected component of $K \cap \overline{B\left(x_{0}, r\right)}$ that contains $x_{0}$. Since there are points of $K$ outside $\overline{B\left(x_{0}, r\right)}$ and $K$ is connected, it is clear that $S$ must touch $\partial B\left(x_{0}, r\right)$; hence $\Gamma:=S \cap \partial B\left(x_{0}, r\right)$ is a finite, nonempty set. We claim that

$$
\mathcal{H}^{1}(S)=\min \left\{\mathcal{H}^{1}(C) \mid C \text { is connected and compact with } \Gamma \cup\left\{x_{0}\right\} \subset C\right\} .
$$

Observe that the $\geq$ sign in place of equality is trivial in (22), since $S$ is connected and compact and $\Gamma \cup\left\{x_{0}\right\} \subset S$. Hence, we assume that

$$
\mathcal{H}^{1}(S)>\min \left\{\mathcal{H}^{1}(C) \mid C \text { is connected and compact with } \Gamma \cup\left\{x_{0}\right\} \subset C\right\}
$$

and seek a contradiction. Indeed, if we set

$$
K^{\prime}=(K \backslash S) \cup C
$$

where $C$ is any set which achieves the minimum in (23), then $K^{\prime}$ is clearly a connected compact set, and $\mathcal{H}^{1}\left(K^{\prime}\right)<\mathcal{H}^{1}(K)$ follows from the assumption that $\mathcal{H}^{1}(C)<\mathcal{H}^{1}(S)$. Moreover, hull $(K)=\operatorname{hull}\left(K^{\prime}\right)$ by construction, since we have not added or removed extreme points. But this is a contradiction, since then we would have

$$
\frac{\mathcal{L}^{2}\left(\operatorname{hull}\left(K^{\prime}\right)\right)}{\mathcal{H}^{1}\left(K^{\prime}\right)^{2}}>\frac{\mathcal{L}^{2}(\operatorname{hull}(K))}{\mathcal{H}^{1}(K)^{2}}=\frac{1}{2 \pi},
$$

thus violating (2). Hence (23) is ruled out, and (22) is necessarily satisfied.

As a consequence, it follows from (22) and Lemma 4.2 below (applied with $P=\Gamma \cup\left\{x_{0}\right\}$ ) that $S$, in particular, contains at least one segment. Hence the tangent space to $K$ is constant along that segment, and thus the direction measure of $K$ has at least one atom. But this is a contradiction, since $\mu$ is a multiple of Lebesgue measure. Hence (21) follows.

Finally, since $K \subset \partial$ hull $(K)$ and $K$ is connected, we obtain that $K$ is homeomorphic either to a segment or to the unit circle. In either case $K$ is a continuous rectifiable curve which maximizes the isoperimetric ratio of the convex hull, hence $K$ is a semicircle (see [10]).

The following lemma follows from the far more general results in 11. However, for the sake of completeness, we present a short self-contained proof in the Appendix.

Lemma 4.2. Let $P$ be a finite subset of $\mathbb{R}^{d}$ consisting of at least two points, and let $S \subset \mathbb{R}^{d}$ be any minimal Steiner network containing $P$, i.e. $S$ is a connected compact set such that $P \subset S$ and

$$
\mathcal{H}^{1}(S)=\min \left\{\mathcal{H}^{1}(S) \mid C \text { is connected and compact with } P \subset C\right\} .
$$

Then $S$ is the union of finitely many segments.

\section{Appendix: Proof of Lemma 4.2}

With the Euclidean metric, $S$ is a compact, connected metric space such that $\mathcal{H}^{1}(S)<\infty$. Then, any two points $x, y \in S$ can be joined by an injective Lipschitz curve with values in $S$ (see e.g. [1, Thm. 4.4.7). Consider two points $x, y \in P$, and let $\gamma_{1}:[0,1] \rightarrow S$ be an injective Lipschitz curve such that

$$
\gamma_{1}(0)=x, \quad \gamma_{1}(0)=y .
$$


Now suppose that, for some $k \geq 1$, we have already constructed $k$ injective Lipschitz curves $\gamma_{i}:[0,1] \rightarrow S, i=1, \ldots, k$, such that

(1) $\gamma_{i}(0) \in P$ for every $i=1, \ldots, k$.

(2) $\gamma_{i}([0,1]) \cap \bigcup_{j<i} \gamma_{j}([0,1])$ consists of the single point $\gamma_{i}(1)$, for every $j=$ $2, \ldots, k$.

Note that this is initially true for $k=1$, since we have already constructed $\gamma_{1}$ (and condition 2 is empty when $k=1)$. If $\bigcup_{i=1}^{k} \gamma_{i}([0,1])$ covers $P$, then stop the process, otherwise take $z_{k} \in P$ not covered by any $\gamma_{i}$ and let $\gamma_{k+1}:[0,1] \rightarrow S$ be an injective Lipschitz curve such that

$$
\gamma_{k+1}(0)=z_{k}, \quad \gamma_{k+1}(1) \in \bigcup_{i=1}^{k} \gamma_{i}([0,1])
$$

and the length of $\gamma_{k+1}$ is a minimum among all curves with these properties. Note that condition 2 is now satisfied also for $i=k+1$, since otherwise one could shorten $\gamma_{k+1}$, which is a contradiction.

Proceeding inductively in this way, the process is stopped in a finite number of steps, since at every new step we cover at least one more point of $P$. Hence, we eventually obtain $k$ curves satisfying conditions 1 and 2 such that

$$
P \subset S^{\prime}:=\bigcup_{i=1}^{k} \gamma_{i}([0,1]) \subseteq S .
$$

Note that $S^{\prime}$ is a connected set by condition 2 ; hence from $P \subset S^{\prime} \subseteq S$ it follows that $S^{\prime}=S$, since $S$ is connected and has minimal length. Then we see that $S$ is the union of $k$ curves satisfying conditions 1 and 2 . If we let

$$
V=P \cup \bigcup_{i=1}^{k}\left\{\gamma_{i}(1)\right\}
$$

we see that, topologically, $S$ is equivalent to the undirected graph $(V, E)$, where $(x, y)$ belongs to the edge set $E$ if and only if the two points $x, y \in V$ are joined by an arc of curve in $S$ which touches no point of $V$ other than $x$ and $y$.

Now, if we remove from $S$ an arc of curve corresponding to an edge $(x, y) \in E$ and replace it with the segment from $x$ to $y$, we reduce the length of $S$ unless this arc is already a segment. Hence, necessarily, $S$ is made up of finitely many segments.

\section{ACKNOWLEDGEMENTS}

The author wishes to thank Michele Gori for fruitful discussions on this problem.

\section{REFERENCES}

[1] L. Ambrosio, P. Tilli, Topics on analysis in metric spaces. Oxford University Press, Oxford, 2004. MR2039660 (2004k:28001)

[2] T. Bonnesen, W. Fenchel, Theory of convex bodies, translated from the German and edited by L. Boron, C. Christenson and B. Smith. BCS Assoc., Moscow, 1987. MR920366 (88j:52001)

[3] H. T. Croft, K. J. Falconer, R.K. Guy, Unsolved problems in geometry. Problem Books in Mathematics. Unsolved Problems in Intuitive Mathematics, II. Springer-Verlag, New York, 1991. MR 1107516 (92c:52001)

[4] E. Egerváry, On the smallest convex cover of a simple arc of space-curve. Publ. Math. Debrecen 1 (1949), 65-70. MR0036021 (12:46e) 
[5] H. Federer, Geometric measure theory. Springer-Verlag, New York, 1969. MR0257325 (41:1976)

[6] M. Gori, On a maximization problem for the convex hull of connected systems of segments. Journal of Convex Analysis 14 (2007), no. 1, 49-68. MR.2310428

[7] M. G. Krĕ̌n, A.A. Nudel'man, The Markov moment problem and extremal problems. Amer. Math. Soc., Providence, R.I., 1977. MR0458081 (56:16284)

[8] Z. A. Melzak, The isoperimetric problem of the convex hull of a closed space curve. Proc. Amer. Math. Soc. 11 (1960), 265-274. MR0116263(22:7058)

[9] Z. A. Melzak, Numerical evaluation of an isoperimetric constant. Math. Comp. 22 (1968), 188-190. MR0223976 (36:7023)

[10] P. A. P. Moran, On a problem of S. Ulam. J. London Math. Soc. 21 (1946), 175-179. MR0020799 (8:597n)

[11] F. Morgan, (M,e,d)-minimal curve regularity. Proc. Amer. Math. Soc. 120 (1994), 677-686. MR.1169884 (94e:49018)

[12] A. A. Nudel'man, Isoperimetric problems for the convex hulls of polygonal lines and curves in multidimensional spaces. (Russian) Mat. Sb. (N.S.) 96(138) (1975), 294-313. MR0375090 $(51: 11286)$

[13] I. J. Schoenberg, An isoperimetric inequality for closed curves convex in even-dimensional Euclidean spaces. Acta Math. 91 (1954), 143-164. MR0065944 (16:508b)

[14] A. Siegel, Some Dido-type inequalities. Elem. Math. 56 (2001), no. 1, 17-20. MR.1818263

[15] A. Siegel, A Dido problem as modernized by Fejes Tóth. Discrete Comput. Geom. 27 (2002), no. 2, 227-238. MR $1880939(2002 \mathrm{~m}: 52009)$

[16] V. A. Zalgaller, Extremal problems on the convex hull of a space curve. (Russian) Algebra $i$ Analiz 8 (1996), no. 3, 1-13. Translation in St. Petersburg Math. J. 8 (1997), no. 3, 369-379. MR:1402285 (97c:58028)

Dipartimento di Matematica, Politecnico di Torino, 10129 Torino, Italy

E-mail address: paolo.tilli@polito.it 